\title{
Éditorial : Situations de violence et migration
}

Marie-Antoinette Hily et Christian Poiret

\section{(2) OpenEdition}

\section{Journals}

Édition électronique

URL : https://journals.openedition.org/remi/14326

DOI : $10.4000 /$ remi. 14326

ISSN : $1777-5418$

Traduction(s) :

Editorial: Situations of Violence and Migration - URL : https://journals.openedition.org/remi/14781 [en]

\section{Éditeur}

Université de Poitiers

\section{Édition imprimée}

Date de publication : 1 octobre 2020

Pagination : 7-9

ISBN : 979-10-90426-66-5

ISSN : 0765-0752

Référence électronique

Marie-Antoinette Hily et Christian Poiret, «Éditorial : Situations de violence et migration », Revue européenne des migrations internationales [En ligne], vol. 36 - $n^{\circ} 1$ | 2020, mis en ligne le 01 octobre 2020, consulté le 14 avril 2022. URL : http://journals.openedition.org/remi/14326 ; DOI : https:// doi.org/10.4000/remi. 14326 


\section{Éditorial: Situations de violence et migration Marie-Antoinette Hily ${ }^{1}$ et Christian Poiret ${ }^{2}$}

Images de colonnes de réfugiés fuyant les zones de combats, images d'un marché aux esclaves en Libye, images de corps rendus par la mer ou jalonnant les pistes du désert, images de harcèlement policiers aux frontières de la forteresse Europe, images des camps et de la violence de l'enfermement, etc., les médias, les intellectuels, les médecins, les humanitaires, les ONGs se chargent de nous rappeler quotidiennement à quel point violences et migrations peuvent avoir partie liée. Et encore, ces images ne nous disent rien des violences domestiques et interpersonnelles plus discrètes, et notamment des violences de genre, des violences sexuelles qui peuvent contribuer à la décision de choisir l'exil ou qui éprouvent et complexifient les parcours migratoires. Elles ne nous disent rien non plus des violences symboliques qui redoublent celles subies en migration quand des politiques migratoires et de l'asile tendent à minimiser, à contester ou à dénier les expériences subies et vécues.

La violence ne peut pas être réduite à des faits objectifs ni probablement appréhendée "objectivement ", elle relève aussi de la perception et de l'expérimentation qu'en ont et qu'en font les acteurs dans des situations et des contextes. Ainsi, un même fait peut-il être ou non caractérisé comme violent selon les normes et les jugements de ceux qui l'évaluent et qui dès lors lui " attribuent valeur ". Et elle ne peut pas être seulement appréhendée " subjectivement " comme un phénomène subi : dans leur rapport à ce qui est vécu comme violence, les migrants élaborent des stratégies ou des tactiques d'évitement, de protection, pour faire face aux dangers. Et comment se dit-elle dans les récits que font les migrants? En vue de quoi ? À destination de qui ? Et dans quels contextes?

Quelques-unes de ces questions abordées dans ce dossier viennent en complément du numéro de la Revue Européenne des Migrations Internationales " Dire la violence des frontières. Mises en mots de la migration vers l'Europe $n^{3}$. Les articles publiés alors s'intéressaient à l'objet frontière interpellé par les discours, ceux des migrants essentiellement qui dans leurs parcours migratoires

\footnotetext{
1 Sociologue, MIGRINTER, Université de Poitiers/CNRS, MSHS, Bât. A5, 5, rue Théodore Lefebvre, TSA 21103, 86073 Poitiers cedex 9 ; marie-antoinette.hily@orange.fr

2 Sociologue, Maître de conférences HDR, URMIS, Université de Paris/CNRS/IRD, casier 7027, 75205 Paris cedex 13 ; poiret@u-paris.fr

3 Canut Cécile, Pian Anaïk et Véronique Petit (Coords.) (2017) Dire la violence des frontières. Mises en mots de la migration vers l'Europe, Revue Européenne des Migrations Internationales, 33 (2-3).
} 
éprouvent la violence des obstacles à affronter ; autant de frontières physiques, sociales, juridiques et politiques qui mettent en danger ceux qui veulent les franchir. L'approche, en privilégiant la production des discours de différents acteurs, venait combler un vide dans la recherche et apportait de nouveaux éléments de compréhension des processus de migration. Ainsi comme le concluaient Cécile Canut et Anaïk Pian dans leur éditorial : " les mises en mots de la violence des frontières et aux frontières offrent [...] une grille de lecture des inégalités sociospatiales à I'heure de la mondialisation " (p. 16).

Si ce dossier nous a bien informés sur les épreuves des migrants et sur leurs récits des violences, les articles que nous publions ici relèvent plus directement d'un questionnement sur l'inhospitalité et sur les violences qui affligent les mobilités des hommes et des femmes que ce soit par voie terrestre ou par voie maritime.

Sans directement rendre compte des nombreux usages du concept de violence qui, même adjectivé, violence structurelle, violence institutionnelle, violence interpersonnelle, violence raciste, sexiste, classiste, violence physique, psychologique, symbolique, recouvre un large champ de significations, au risque d'ailleurs de dilution du sens, les auteurs publiés dans ce volume ont contribué aux débats en revenant plus directement sur les pratiques et les étapes des carrières migratoires. On retrouvera les problématiques d'entrée en migration, les aléas des routes empruntées et les situations plus ou moins durables d'installation. On retrouvera également la question centrale du rôle de l'État et des logiques administratives et de leurs conséquences sur les populations migrantes.

L'article de I'historien Yann Scioldo-Zürcher qui ouvre le dossier " situations de violence et migration " a pour objet le rôle politique de l'État d'Israël dans la mise en œuvre de logiques administratives organisant la migration et l'installation de populations juives. L'auteur montre que dès le début des années 1950, l'Agence juive participe activement à organiser les transferts puis l'installation des migrants en leur imposant une "violence administrative légitime ". La sélection et la classification des migrants, l'organisation des départs et le voyage, la répartition dans l'espace national, toutes ces étapes font l'objet d'un ensemble de réglementations qui devaient servir la construction de la nation israélienne, au détriment des situations individuelles et au seul profit de l'édification d'un pays "à la fois compris comme un "État juif" et un "État pour les Juifs" ". L'expérience migratoire sioniste de la décennie 1950, que I'auteur examine en la débarrassant de tout point de vue moral, ouvre sur la question de la normalisation sociale, qui se présente dans le cas d'Israël au cours des années suivant la reconnaissance de l'État, comme la condition de réussite d'un projet national dans une volonté qui répond à l'urgence d'une situation historique.

Laurent Faret décrit une autre situation où la violence est liée aux trafics de drogue, aux inégalités sociales, à la pauvreté inscrite dans le fonctionnement des formations sociales. II situe ses enquêtes dans le Mexique des années 2010 où les "populations en mobilité " sont particulièrement vulnérables à la merci des groupes criminels ou des forces de l'ordre. L'auteur s'intéresse aux dispositifs étatiques qui renforcent les contrôles des populations migrantes avec notamment le plan répressif "Frontera Sur » (juillet 2014) qui vise les migrants centraméricains voulant entrer aux États-Unis. Violence d'État et violence 
criminelle, il y a là un double danger pour ces migrants qui s'engagent dans un périple où domine l'incertitude et la peur, mais aussi la volonté de faire aboutir le voyage à tout prix.

Les réponses des migrants aux politiques de deux États, États-Unis et Mexique, sont décrites dans I'article d'Olga Odgers-Ortiz. Arrêtées par le mur, les populations migrantes sont contraintes de choisir entre une installation au Mexique en attendant de pouvoir partir, un passage clandestin qui constitue une solution à haut risque, ou " une redéfinition de leur projet migratoire " en se sédentarisant dans une ville mexicaine. Pour ceux qui parviennent à atteindre la frontière, les épreuves qu'ils ont endurées tout au long de leur parcours sont relativisées au point d'être présentées comme le "prix à payer». De l'autre côté du mur, ils disent être déconcertés par la violence de la procédure de demande d'asile, violence qu'ils ne soupçonnaient pas. On peut penser aussi que se résigner est une autre forme de violence qui a son prix, celui payé très cher de courber l'échine devant des logiques de fermeture des frontières.

Émilie Adam-Vézina met au jour l'expérience de la violence que font des femmes d'Afrique subsaharienne installées en France et vivant avec le $\mathrm{VIH}$. Elle explore l'imbrication du vécu des violences, de la maladie et de la migration et son effet sur les trajectoires de ces femmes. À l'aide de données empiriques recueillies dans le cadre d'une recherche doctorale, elle montre que l'articulation de ces trois composantes est révélatrice des processus sociaux qui structurent leurs trajectoires sociales et migratoires. Restituer l'expérience de violences permet d'en montrer les effets sur leur projet migratoire et leur état de santé. Car si les liens entre la migration et l'état de santé sont mieux connus, l'article traite d'une dimension encore peu étudiée, celle de l'articulation complexe entre un état de santé altéré par le VIH-sida, l'expérience migratoire et le vécu de violences. L'auteure distingue trois formes de violence, que vivent ces femmes, généralisée, institutionnelle et interpersonnelle, qui se manifestent aux différentes étapes des parcours migratoires : celles subies dans le pays d'origine, qui motivent leur départ, puis sur les routes où elles aggravent leur vulnérabilité aux risques de contamination et enfin en France où elles accentuent leur précarité et renforcent I'incertitude quant à l'acquisition des droits de séjour.

La chronique juridique de Oriana Philippe "I'arme juridique aux confins de la France et de I'Italie " montre comment l'État français utilise le Code frontières Schengen pour pratiquer des " contournements normatifs" selon les situations. Par exemple en 2015 après I'attaque terroriste, la France va - avec la mise en place de l'état d'urgence - officialiser la pratique des contrôles aux frontières nationales. Face à ces contournements qui entravent le principe de libre circulation dans l'espace Schengen et alors que la solidarité se renforce aux confins franco-italiens, avec la condamnation de Cédric Herrou notamment, la riposte des associations de défense va se faire justement par la mobilisation du droit. La maitrise accrue du droit dans l'affrontement avec l'État devient en effet une arme qui de défensive devient offensive en se perfectionnant dans les actions juridiques.

Ainsi certaines institutions étatiques apparaissent, dans les articles de ce dossier, comme des acteurs majeurs des processus de construction des " mondes violents " que rencontrent les migrants dans leurs parcours migratoires. 